EXTENDED REPORT

\title{
Deep lamellar keratoplasty by deep parenchyma detachment from the corneal limbs
}

\author{
T Senoo, K Chiba, O Terada, J Mori, M Kusama, K Hasegawa, Y Obara
}

See end of article for

authors' affiliations

Br J Ophthalmol 2005;89:1597-1600. doi: 10.1136/bjo.2005.072215

................. Aim: To improve the deep lamellar keratoplasty technique.

Correspondence to: Tadashi Senoo, 3210293, 880 Kitakobayashi Mibu, Tochigi/Japan, Dokkyo University School of Medicine, Department of Ophthalmology, Tochigi, Japan; senoo@ dokkyomed.ac.jp

Method: For the easy and reliable perfomance of deep lamellar keratoplasty (DLKP), detachment of Descemet's membrane through the corneal limber flap was improved. To expose Descemet's membrane, the parenchyma was detached by hydrodelamination through a sclerocorneal flap made in the corneal limbs. The parenchyma was removed after the pseudochamber between it and Descemet's membrane was maintained with viscoelastic material. The corneal graft was placed with a running suture. 22 eyes were treated.

Results: Complete exposure of Descemet's membrane was obtained in 20 of the 22 eyes (91\%). The membrane was perforated in five of the 22 eyes (23\%) during surgery, and two of the 22 eyes (9\%) were converted to penetrating keratoplasty. These two eyes developed keratoconus after acute corneal hydrops.

Accepted for publication 1 July 2005

Conclusion: Compared with the conventional procedure, this new method provides easy, reliable exposure of Descemet's membrane.

$\mathrm{D}$ eep lamellar keratoplasty (DLKP), performed while the corneal endothelial function is maintained, is reported to provide a good sense of vision, comparable to that obtained by penetrating keratoplasty (PKP). This type of surgery is advantageous in that there is no endothelial rejection because the host's endothelium remains intact and does not affect the endothelial condition of the donor cornea. Moreover, even a stored cornea can be used. ${ }^{1-4}$ DLKP, however, has not been used widely because the surgical method is more complex than that for PKP. In previously published studies, air injection and viscodelamination and hydrodelamination between Descemet's membrane and the parenchyma have been used for membrane detachment, ${ }^{15}$ but with many of the reported techniques it is difficult to completely expose Descemet's membrane. To overcome this drawback we have developed a new DLKP surgical method, in which Descemet's membrane is exposed quickly, completely, and safely. We here report the use of this improved surgical procedure.

\section{MATERIALS AND METHODS \\ Patients}

Twenty two eyes of 21 patients ( 11 men, 10 women; average age 59.1 (SD 20) years) treated by this new method were observed for more than 6 months. The original diseases were corneal leucoma (eight), keratoconus (six), herpetic keratitis (three), macular dystrophy (two), granular dystrophy (one), lattice dystrophy (one), and corneal chemical burn (one). Sixty seven eyes of 61 patients ( 25 men, 36 women; average age 57.5 (20.8) years) were operated on by the conventional method, performed according to Sugita's method. ${ }^{1}$ The original diseases were corneal leucoma (25), keratoconus (16), herpetic keratitis (eight), macular dystrophy (one), granular dystrophy (seven), lattice dystrophy (three), corneal chemical burn (three), Stevens-Johnson syndrome (two), and Schneider dystrophy (two). No difference in surgical indications was found between the two groups. Comparisons were made between the groups on complications during and after surgery, and on visual acuity and corneal endothelial cell density. Patients with other associated diseases of visual disturbance and those whose eyes were converted to PKP

were excluded from the evaluation of visual acuity. Data were analysed statistically by the $\chi^{2}$ and Student's $t$ tests.

\section{Surgical technique}

A sclerocorneal flap, as in trabeculectomy, is made at the 1011 o'clock position, exposing the deep parenchyma of the corneal limbs until Schlemm's canal is reached. Part of Descemet's membrane is exposed by making this flap. After marking the range of removal of the parenchyma with a trephine, the aqueous humour is exchanged for air through a side port. This side port should be as close as possible to the corneal limbs. If it is made near the corneal centre, there is the possibility of Descemet's membrane perforation and hydrodelamination of the membrane from the port. Next, Descemet's membrane is partially separated from the posterior parenchyma by a spatula inserted through the flap. It can be inserted into the deep corneal parenchyma without resistance (fig 1). Descemet's membrane then is separated completely from the posterior parenchyma by hydrodelamination at the point of detachment by the spatula. The membrane is pushed down by the flow of air to the anterior chamber (fig 2). Through the flap, a viscoelastic material is injected between the separated membrane and parenchyma. At this point, it should be confirmed by means of the compressed air position that the area of the separated Descemet's membrane is larger than the removed area (fig 3). The corneal parenchyma then is incised with a Barron aspiration trephine until the viscoelastic material flows out, after which Descemet's membrane is exposed (figs 4, 5). After stripping off the donor Descemet's membrane, the full thickness donor corneal button is sutured into the recipient bed (fig 6).

\section{RESULTS}

Table 1 compares the conventional and new methods. Surgery took 94 (SD 25.7) minutes by the conventional method and 59 (26.1) minutes by the new one, a statistically significant difference $(\mathrm{p}<0.0001)$. Intraoperative Descemet's

Abbreviations: DF, dispersion factor; DLKP, deep lamellar keratoplasty; PKP, penetrating keratoplasty 


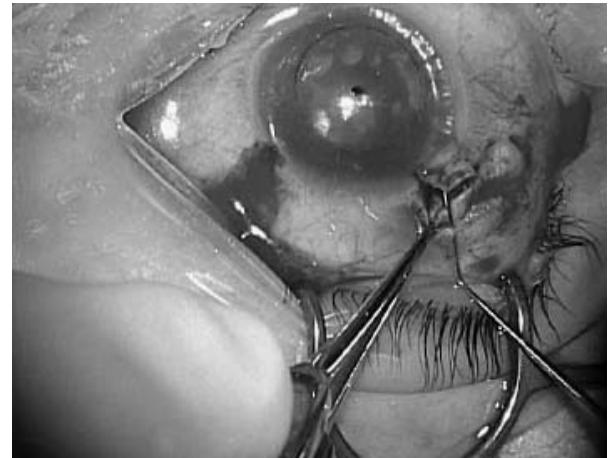

Figure 1 A spatula is inserted from the sclerocorneal flap, and a pocket for hydrodelamination made in the space between Descemet's membrane and the parenchyma.

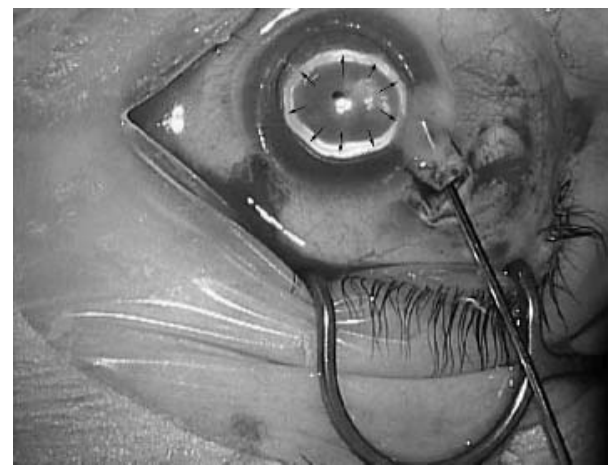

Figure 2 Hydrodelamination is performed through the sclerocorneal flap. Arrows indicate the intra-anterior chamber air. In this figure, Descemet's membrane is pushed down by the flow of air to the anterior chamber.

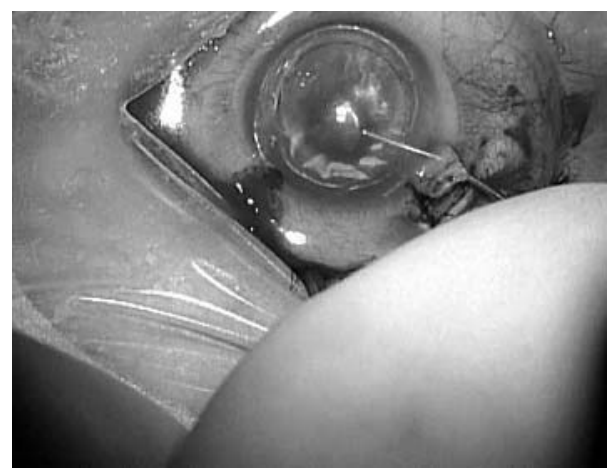

Figure 3 Injection of viscoelastic material. After injection, the area of the detached Descemet's membrane must be confirmed to be wider than the marker of the removal area.

membrane perforation occurred in five eyes $(22.7 \%)$ with the new method and in 25 eyes $(37.3 \%)$ with the conventional one. There was no statistical difference between the two groups $(\mathrm{p}=0.21)$. Conversion to PKP during surgery occurred for two eyes (9\%) with the new method and nine eyes $(13 \%)$ with the conventional one. The two eyes, for which there was conversion to PKP, developed keratoconus after acute corneal hydrops. In those two cases, detachment of Descemet's membrane by hydrodelamination was difficult, and forced injection of viscoelastic material between the membrane and parenchyma caused widespread tears in the

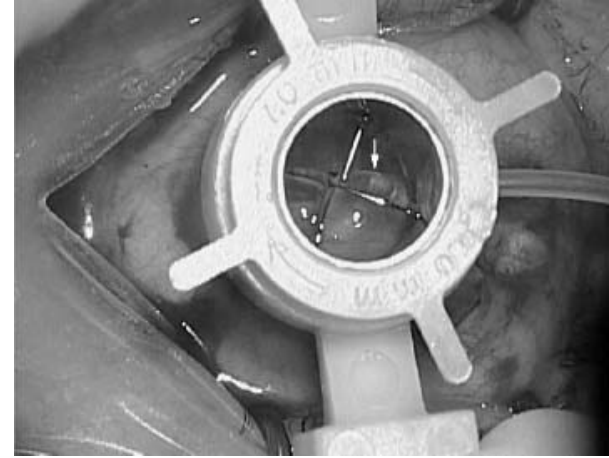

Figure 4 Corneal perforation by a vacuum trephine. On puncture, the viscoelastic material flows out of the perforation (arrow).

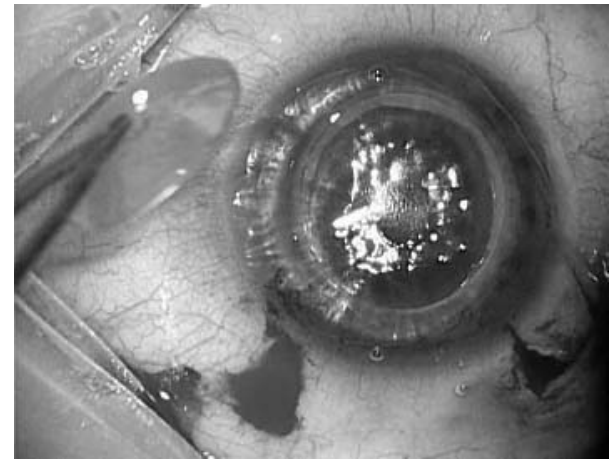

Figure 5 Descemet's membrane is exposed completely in the transplantation area.

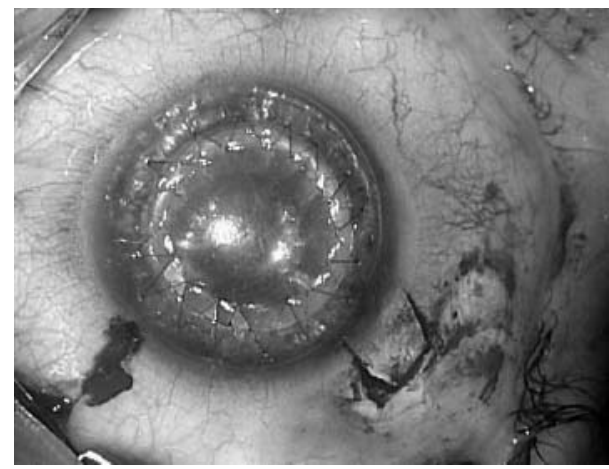

Figure 6 A full thickness donor corneal button lacking Descemet's membrane is sutured to the recipient bed.

membrane, rendering it unrepairable. A double anterior chamber was present after surgery in 17 (29.3\%) of 58 eyes with the conventional method and in nine (45\%) of 20 eyes with the new one. There was no significant difference between the two groups $(p=0.16)$. The mean duration of the postoperative double anterior chamber was 1.5 (2.5) days (range 0-10 days) with our method and 4.7 (16.7) days (range 0-120 days) with the conventional one, and there was a significant difference between the methods $(p=0.038)$. Because of a postoperative double anterior chamber in three eyes $(5.2 \%)$ PKP was performed by the conventional method and in none by the new method. The mean postoperative, best corrected visual acuity was 0.55 (dispersion factor (DF): $1.93, \mathrm{n}=45$ ) for the conventional method and 0.73 (DF: 1.34 , 
Table 1 Comparison of the conventional and new methods

\begin{tabular}{lll}
\hline & New method & Conventional method \\
\hline Duration of surgery (minutes) & $59(26.1)^{*}(n=22)$ & $94(25.7)^{*}(n=67)$ \\
Intraoperative Descemet's membrane perforation & $5(22.7 \%)(n=22)$ & $25(37.3 \%)(n=67)$ \\
Conversion to PKP (eyes) & $2(9 \%)(n=22)$ & $25(37.3 \%)(n=67)$ \\
Postoperative double anterior chamber (eyes) & $17(29.3 \%)(n=20)$ & $9(45 \%)(n=58)$ \\
Duration of the postoperative double anterior & $1.5(2.5)^{*}(n=20)$ & $4.7(16.7)^{*}(n=58)$ \\
chamber (days) & & \\
Postoperative best corrected visual acuity & $0.73(\mathrm{DF} ; 1.34)^{*}(n=18)$ & $0.18(\mathrm{DF}: 2.44)^{*}(n=45)$ \\
Postoperative corrected visual acuity 1 month later & $0.70(\mathrm{DF}: 0.30)^{*}(n=18)$ & $0.18(\mathrm{DF}: 2.44)^{*}(n=45)$ \\
Endothelial cell density at 1 year (cells $\left./ \mathrm{mm}^{2}\right)$ & $1722.8(665.3)(n=18)$ & $2044.8(678.2)(n=45)$ \\
\hline${ }^{*} p<0.05$ between groups. & & \\
\hline
\end{tabular}

$\mathrm{n}=18$ ) for the new one. The difference between the groups was significant $(p=0.039)$. The mean postoperative corrected visual acuity 1 month later was 0.18 (DF: 2.44, $\mathrm{n}=45$ ) for the conventional method and 0.70 (DF: $0.30, n=18$ ) for the new one, the difference between the groups being statistically significant $(\mathrm{p}<0.0001)$. Mean corneal endothelial cell density at 1 year was 2044.8 (678.2) cells $/ \mathrm{mm}^{2}$ for the conventional method and 1722.8 (665.3) cells $/ \mathrm{mm}^{2}$ for the new method; this difference between the groups was not significant $(\mathrm{p}=0.15)$.

\section{CASE REPORT}

Macular corneal dystrophy was diagnosed in a 38 year old man, and this type of operation indicated. (The surgical findings for this patient are shown in a paragraph under "surgical technique".) His preoperative visual acuity was 0.4, and intraocular pressure $12 \mathrm{~mm} \mathrm{Hg}$. Corneal endothelial cell density was not measured for corneal opacity. Surgery was performed without complications in a period of 34 minutes. Figure 7 shows photographs taken before and after surgery. His postoperative visual acuity was 1.2, intraocular pressure $13 \mathrm{~mm} \mathrm{Hg}$, and corneal endothelial cell density 2500 cells/ $\mathrm{mm}^{2}$.

\section{DISCUSSION}

Deep lamellar keratoplasty has certain advantages over penetrating keratoplasty for many patients who retain endothelial function because endothelial rejection is not expected and postoperative visual acuity is equal to that under PKP. Furthermore, as there is no need for a graft to obtain endothelial cells, a stored donor cornea can be used..$^{1-4}$

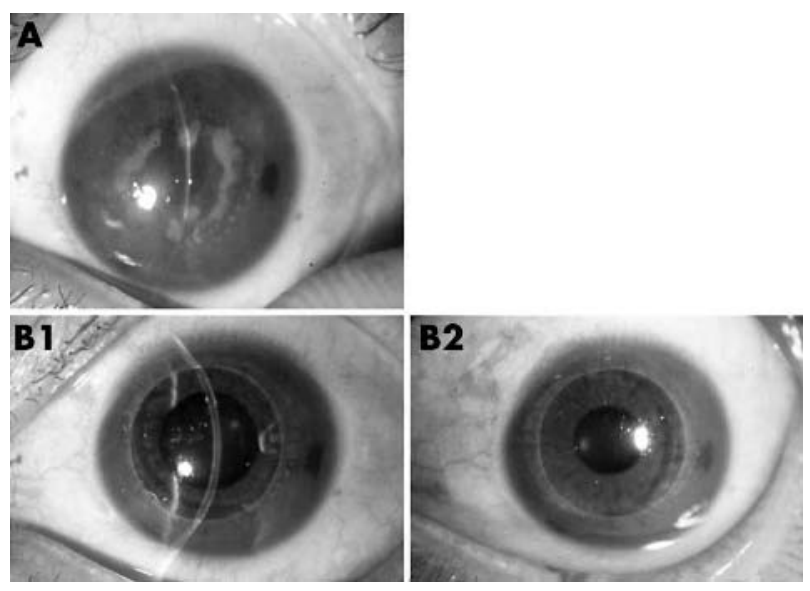

Figure 7 Case: Corneal macular dystrophy. Preoperative visual acuity was 0.4 , postoperative visual acuity 1.2. Postoperative endothelial cell density was 2500 cells $/ \mathrm{mm}^{2}$. (A) Preoperative finding. (B) Postoperative findings.
Although advantageous, this procedure has not been widely used because the surgical technique is more difficult than that for PKP. We here reported a novel method of detaching Descemet's membrane through a corneal limber flap, thereby facilitating deep lamellar keratoplasty. Our surgical method, developed from that of Sasaki et al, ${ }^{6}$ exposes Descemet's membrane through a limbal flap. After exposure, Descemet's membrane is detached by hydrodelamination. Surgeons who have experience with DLKP and trabeculectomy can easily understand and use this method. Our findings showed little difference in the intraoperative complications associated with this and the conventional method and that conversion to PKP during surgery was infrequent, except in special cases. Detachment of Descemet's membrane was easy in patients who did not have adhesion of the membrane and pathological parenchyma. In those who presumably had such adhesion, forced injection of viscoelastic material used in hydrodelamination caused cracking of the membrane. In cases in which there is possible adhesion of Descemet's membrane to the corneal parenchyma, as when there is keratoconus after acute corneal hydrops, the membrane must be very carefully detached.

A postoperative double anterior chamber tended to form with greater frequency under this new method, but it soon disappeared because there was no remaining corneal parenchyma unlike under the conventional method. With this new method, a PKP reoperation caused by a postoperative double anterior chamber was needed. This is because the corneal parenchyma had been excised completely. With the conventional method, there were three cases of further PKP surgery.

As for mean postoperative best corrected visual acuity and mean postoperative visual acuity 1 month after surgery, the new method was better statistically than the conventional one, the mean postoperative corrected visual acuity at 1 month being 0.18 for the conventional method and 0.70 for the new one. Other reports have suggested that the cause of postoperative low visual acuity is scarring that occurs at the interface between the donor cornea and parenchyma of the recipient cornea. ${ }^{1}$ Because histological reconstruction can be done early, owing to complete Descemet's membrane exposure by our new method, there is early visual acuity.

Melles et al reported a method used in a cataract operation whereby Descemet's membrane was detached by direct injection of a viscoelastic material after making a sclerocorneal tunnel. ${ }^{6-9}$ The differences between their method and ours are: (1) our new method uses trabeculectomy to detach Descemet's membrane, whereas Melles et al used an incision, as in cataract surgery; (2) a flap is made, as in trabeculectomy, and the region directly above Descemet's membrane is reached under direct vision in the new method, whereas Melles et al used air replacement in the anterior chamber and a mirror image at the tip of a 30 gauge needle in order to be directly above Descemet's membrane from the corneal 
parenchyma; (3) in our new method, Descemet's membrane is detached by hydrodelamination and viscoelastic material used to maintain the space between the membrane and corneal parenchyma, whereas Melles et al detached the membrane by means of viscoelastic materials. This difference ensures easy, reliable exposure of Descemet's membrane for the following reasons. With the trabeculectomy-like approach used with the new method, the depth near Descemet's membrane can be confirmed under direct vision, the procedure can be done with smaller incisions, and surgeons with experience in trabeculectomy can easily recognise the exposure image of Schlemm's canal. When there is severe corneal opacity, obtaining mirror images is difficult by the method of Melles et al. If exposure of Schlemm's canal or of Descemet's membrane can be confirmed, subsequent surgical procedures can be done more surely. In our experience, visual confirmation under a microscope is essential for the success of this type of surgery, providing a wider range of indications and reliable surgical results. In eyes without Descemet's membrane adhesion, there was no difference between use of the method of Melles et al and the new one. The former, without hydrodelamination, resulted in a faster surgical procedure. When, however, there was adhesion between Descemet's membrane and the corneal parenchyma large cracks in the membrane tended to occur when it was detached by viscoelastic material. Once the membrane is cracked, the viscoelastic material may enter the anterior chamber, making repair difficult. These findings indicate that forced detachment of Descemet's membrane by the use of viscoelastic material should be avoided when possible. In this respect, our new procedure is considered a relatively safer means by which Descemet's membrane is detached gently by hydrodelamination. Surgery can be done after confirming the presence or absence of Descemet's membrane adhesion.

A drawback of our new method that requires further study is the sharp rise in intraocular pressure because the membrane is exposed over the entire range of resection. Another complication is that carelessness in suturing the donor corneal flap is apt to cause cracks in the membrane. Actually, of the three cases of Descemet's membrane perforation (excluding the two cases of keratoconus with Descemet's membrane adhesion), two were the result of membrane cracking as a result of a rise in intraocular pressure from the side port caused by air replacement after membrane exposure. The other was a case of Descemet membrane perforation by a needle during suturing of the donor corneal flap. At present, we see to it that the graft diameter is shortened $(7.0-7.25 \mathrm{~mm})$ and that a small side port is made. Problems that occur because of the complete exposure of Descemet's membrane, however, need further study.

\section{Authors' affiliations}

T Senoo, K Chiba, O Terada, J Mori, M Kusama, Y Obara, Dokkyo

University School of Medicine, Department of Ophthalmology, Japan

K Hasegawa, International University of Health and Welfare, Japan

\section{REFERENCES}

1 Sugita J, Kondo J. Deep lamellar keratoplasty with complete removal of pathological parenchyma for vision improvement. $\mathrm{Br} J$ Ophthalmol 1997;81:184-8.

2 Morrison JC, Swan KC. Full-thickness lamellar keratoplasty. A histologic study in human eyes. Ophthalmology 1982;89:715-19.

3 Panda A, Bageshwer LM, Ray M. Deep lamellar keratoplasty versus penetrating keratoplasty for corneal lesions. Cornea 1999;18:172-175.

4 Krumeich JH, Daniel J. Depth of lamellar keratoplasty with the guided trephine system for transplantation of full-thickness donor sections. Ophthalmologe 1998; 11:748-54.

5 Sasaki K, Shikatani Y. Exposure of Descemet membrane through corneal limbal approach in deep lamellar keratoplasty. Jap J Clin Ophthalmol 2001;55:297-9.

6 Melles GR, Lander F, Rietveld F, et al. A new surgical technique for deep parenchymal, anterior lamellar keratoplasty. $\mathrm{Br} J$ Ophthalmol 1999;83:327-33

7 Melles GR, Remeijer L, Geerards AJ, et al. A quick surgical technique for deep anterior lamellar keratoplasty using visco-dissection. Cornea 2000;19:427-32.

8 Melles GR, Rietveld FJ, Beekhuis WH, et al. A technique to visualize corneal incision and lamellar dissection depth during surgery. Cornea 1999;18:80-6. 9 Melles GR, Remeijer L, Geerards AJ, et al. The future of lamellar keratoplasty. Curr Opin Ophthalmol 1999;10:253-9. 